\title{
Enfrentamiento a un tumor de peñazco: estudio y posibilidad diagnóstica ${ }^{1}$
}

\section{Facing a petrous tumor: Study and diagnostic possibility}

\author{
Jorge Caro $\mathrm{L}^{2}$, Marcela Castillo $\mathrm{F}^{3}$, Andrés Finkelstein $\mathrm{K}^{3}$.
}

\begin{abstract}
RESUMEN
Los tumores de punta de peñasco se pueden presentar con hipoacusia sensorioneural y un síndrome vertiginoso secundario a hidrops endolinfático. Dentro del diagnóstico diferencial se encuentran los tumores de saco endolinfático, los cuales han sido reportados cada vez con mayor frecuencia en la literatura.

A continuación informamos el caso de un paciente de 50 años que se presenta a la consulta con hipoacusia sensorioneural fluctuante de oído derecho y síndrome vertiginoso. Las neuraimágenes muestran tumor de punta de peñasco que ha tenido un crecimiento progresivo en el tiempo erosionando secundariamente estructuras del oído interno. Se realizó biopsia que no fue concluyente, la cual no ha podido ser repetida por decisión personal de la paciente, por lo que se ha mantenido en manejo expectante.

Dada la evolución y las imágenes se plantea diagnóstico de tumor de saco endolinfático, a la espera de una biopsia confirmatoria.

Palabras claves: Tumor saco endolinfático, tumores de punta de peñasco, tumores de oído, hídrops endolinfático.
\end{abstract}

\section{SUMMARY}

The petrous point tumors may appear with sensoneuronal hypoacousia and a vertiginous syndrome secondary to endolymphatic hidrops. Within the differential diagnosis there are the tumors of the endolymphatic sack, which have been reported more and more frequently in the literature.

Below we report the case of a 50 year old patient who arrives to consult with fluctuating sensoneuronal hypoacousia of the right ear and vertiginous syndrome. The neuroimages show a petrous point tumor that has had a progressive growth in time, secondarily eroding structures of the inner ear. A biopsy was taken but it was not conclusive, and in has not been repeated due to the personal decision of the patient, whereby it has been maintained under expectant handling.

\footnotetext{
${ }^{1}$ Trabajo presentado en la Reunión de la Sociedad Chilena de Otorrinolaringología, Medicina y Girugía de Cabeza y Cuello, Mayo 2005.

${ }^{2}$ Profesor Asociado Unidad Docente Académica Otorrinolaringología, Hospital Cínico Pontificia Universidad Católica de Chile.

${ }^{3}$ Médico Grujano, Hospital Cínico Pontificia Universidad Católica de Chile.
} 
Given the evolution and the images, a diagnosis of endolymphatic sack tumor is considered, while awaiting a confirmation biopsy.

Key words: endolymphatic sack tumor, petrous point tumors, ear tumors, hidrops endolymphatic.

\section{INTRODUCCIÓN}

Los tumores de saco endolinfático (TSE) son tumores adenomatosos del hueso temporal con un patrón papilar característico, que han sido clásicamente mal diagnosticados como papilomas del plexo coroideo, adenomas o adenocarcinomas de oído medio. A pesar de su histología benigna, frecuentemente presentan crecimiento lento pero invasivo con importante compromiso local, erosionando y destruyendo el hueso temporal. Esta patología tiene una importante relación con la enfermedad de Von Hippel-Lindau (VHL), enfermedad genética caracterizada por angiomas retinales, hemangioblastomas del sistema nervioso central, feocromocitoma, tumores renales y pancreáticos, y quistes benignos en otros órganos, originada por una mutación de un gen supresor de tumores codificado en el cromosoma 3p25. Los TSE se asocian a $\mathrm{VHL}$ en un $15 \%$ de los casos aproximadamente $e^{1-3,5}$.

LOS TSE se presentan en un amplio rango de edades, diagnosticándose generalmente entre los 20 y 60 años, siendo más precoces y prevalentes en mujeres en los casos asociados a $\mathrm{VHL}^{1}$. A continuación se presenta un caso clínico de un tumor de punta de peñasco que probablemente corresponde a un Tumor de Saco Endolinfático, estando aún la incógnita acerca del diagnóstico definitivo dada la opción personal del paciente de optar por un manejo expectante.

\section{CASO CLÍNICO}

Paciente de 50 años de edad, sexo femenino, con antecedentes de estar en tratamiento por un nódu- lo tiroideo (con levotiroxina) y un síndrome depresivo, consulta en noviembre de 2000 por un cuadro de 2 años de evolución de hipoacusia de oído derecho fluctuante (llegando a ser severa), tinnitus fluctuante, vértigo, cefalea ocasional, diplopia, fotopsias y paresia de mano derecha. Al examen físico y otorrinolaringológico no se encuentra ninguna alteración. Se le realiza una audiometría que muestra hipoacusia mixta del oído derecho con una vía ósea en 40 db (Figura 1). Paciente trae una TAC del año anterior que mostraba una lesión a derecha de $12 \mathrm{~mm}$ en borde posterior de peñasco con densidad de partes blandas (Figura 2), la cual en ese mismo momento había sido complementada con una RNM que mostró un nódulo hiperintenso en T2 en pared posterior de peñasco derecho. Con diagnóstico de granuloma de colesterol que ha producido un hidrops endolinfático, la paciente pierde los controles y reaparece en agosto de 2004. Los síntomas son semejantes, pero al examen físico se aprecia una lesión de coloración azulada muy nítida en la caja timpánica. Se solicita nuevo estudio con TAC que revela una lesión de peñasco mayor a la anterior que compromete el foramen yugular y oído medio, sugerente de paraganglioma (Figura 3). RNM realizada en noviembre de 2004 revela aumento volumétrico con respecto a RNM de 1999, dimensiones de $18 \times 25 \times 24 \mathrm{~mm}$, con compresión sobre el golfo yugular derecho (Figura 4).

Con estos antecedentes, se realiza biopsia en enero de 2005 por vía endoaural, revelando un tejido de aspecto polipoídeo localizado en el hipotímpano en región posterior, el cual fue extraído completamente sin hemorragia secundaria. EStudio anatomopatológico revela un tejido de aspecto inflamatorio sin elementos de paragan- 


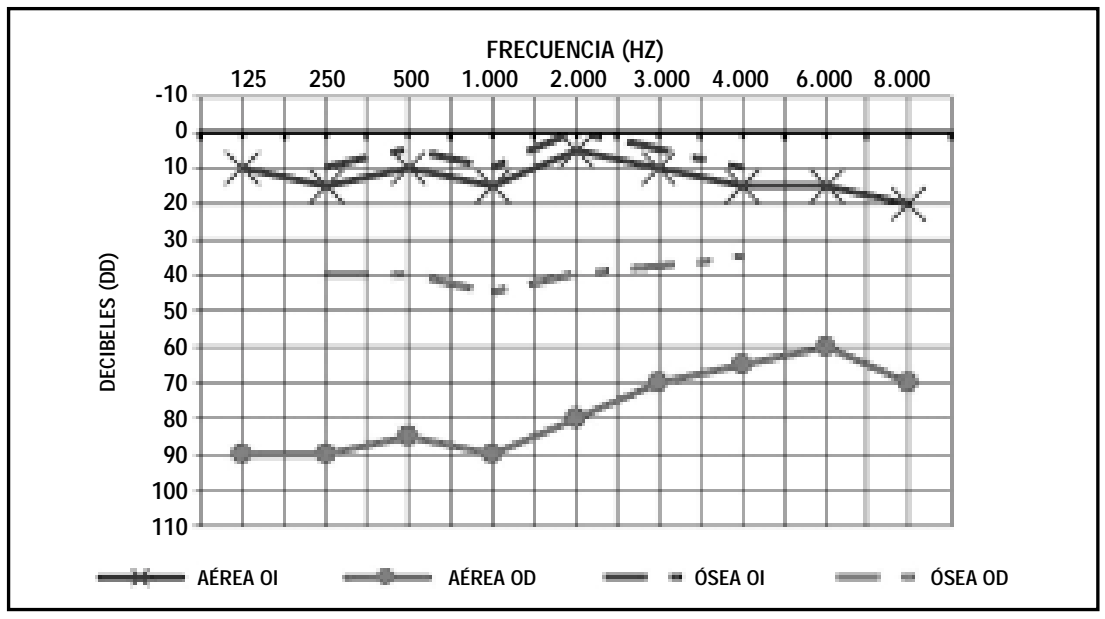

Figura 1. Audiometría

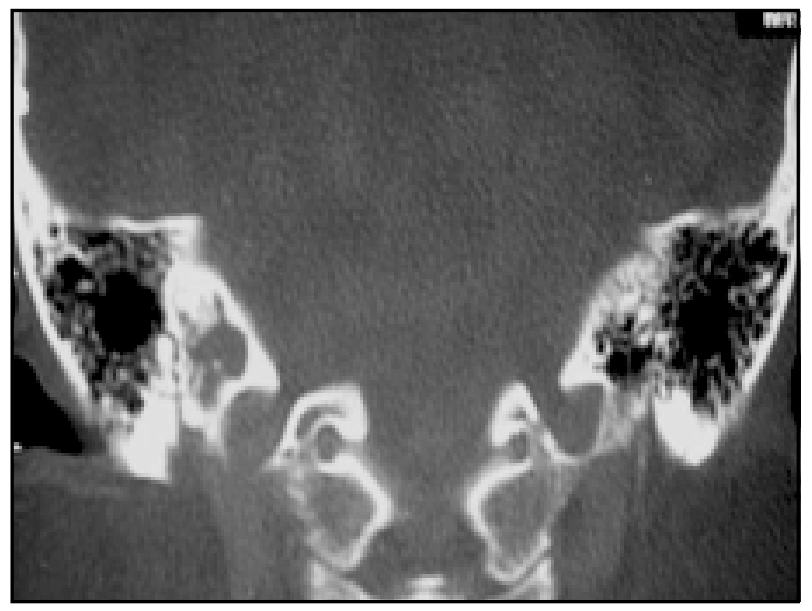

Figura 2. Tomografía computada de oídos, corte coronal.

glioma ni de carcinoma. Se decide repetir RNM en mayo de 2005, revelando masa de peñasco derecho hiperintensa en T2 y RLAIR, sin gran variación con respecto a RNM anterior, midiendo $27 \times 18$ $\mathrm{mm}$ en sus ejes mayores, ubicada por arriba y adelante del golfo de vena yugular. Llega al CAl asomándose al poro acústico desde posterior, asociándose a erosión del conducto coclear y de la pared posterior del peñasco, ocupando parcial- mente la cisterna del ángulo pontocerebeloso, protruyendo a la cavidad del oído medio en estre cho contacto con el canal posterior (Figuras 5A, $5 B$,$) .$

Dada esta evolución, se plantea diagnóstico de tumor de saco endolinfático, por lo que se le propone una nueva biopsia a la paciente, quien la rechaza. Actualmente se mantiene en observación en conjunto con el equipo de neurocirugía. 


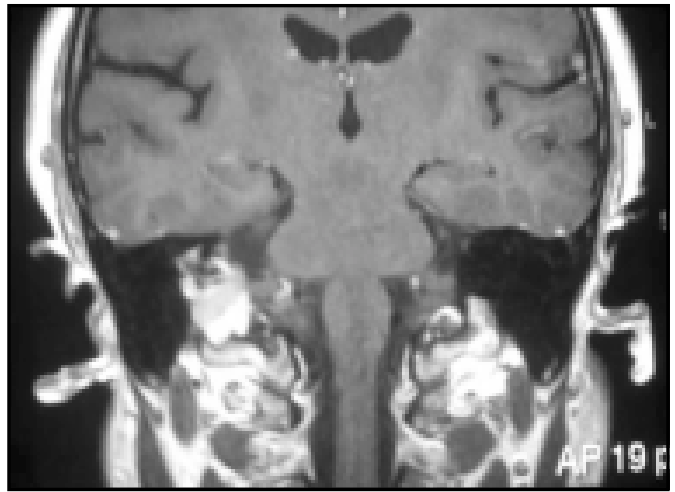

Figura 3. Tomografía computada de oídos, corte coronal.

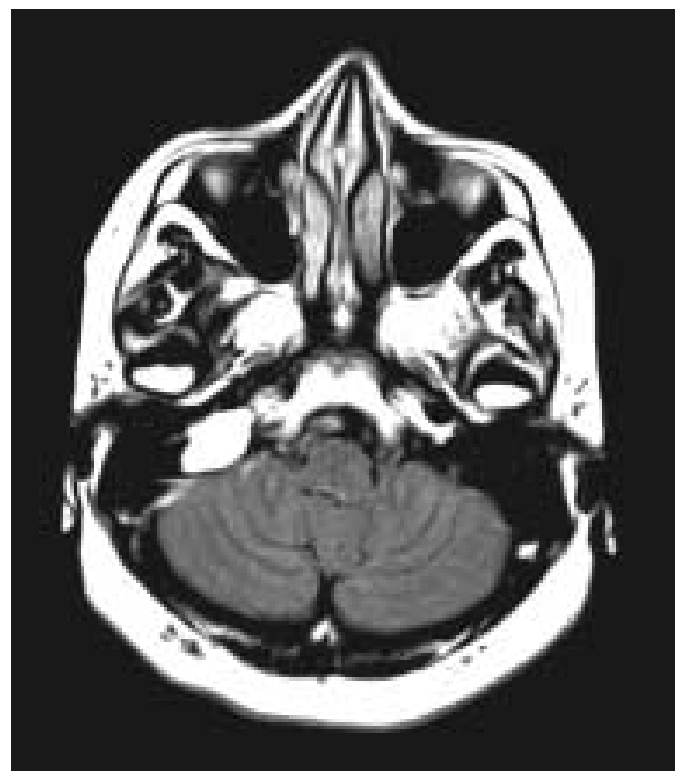

Figura 5. Resonancia nuclear magnética de oídos, corte coronal T2.

\section{DISCUSIÓN}

Los TSEson un tipo de tumor poco frecuente, pero que cada vez han sido reportados con más frecuencia en la literatura, probablemente debido a la mayor preocupa ción einterés por parte de los clínicos para reconocerlo como una entidad propia, sumado al aumento de los recursos técnicos necesarios para su diagnóstico.

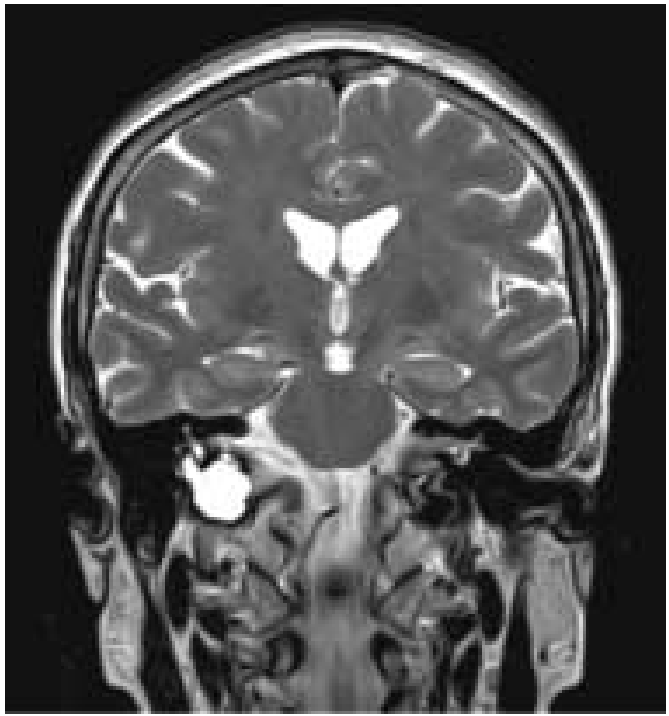

Figura 4. Resonancia nuclear magnética de oídos.

Desdeel punto de vista histológico, son tumores altamente vascularizados, polipoídeos, con áreas quísticas, que característicamente muestran crecimiento papilar con importante destrucción de tejidos blandos y hueso. Es infrecuente encontrar mitosis. La inmunohistoquímica muestra actividad importante para $S 100$ y citoqueratina, siendo las tinciones para membrana epitelial y vimentina también positivas en menor magnitud. En el caso clínico presentado, a pesar de la exploración de la caja timpánica, sólo se pudo extraer un tejido polipoídeo, que en el intraoperatorio impresionó como parte de la lesión en estudio. Lamentablemente, no fue repre sentativa de la lesión, correspondiendo sólo a una porción inflamatoria perilesional que no aportó al diagnóstico definitivo de la patología del paciente'.

Las manifestaciones clínicas de los TSE van a depender principalmente del tamaño que presente el tumor. La hipoacusia sensorioneural es generalmente el primer síntoma en aparecer, siendo también el más frecuente. Su origen probablemente corresponde a la obstrucción del ducto endolinfático con el consecuente hidrops y destrucción del órgano de Corti. En general la hipoacusia es de comienzo insidioso, pero se han descrito casos de presentación brusca. A medida 
que el tumor crece y las estructuras laberínticas son comprometidas, los pacientes comienzan a sufrir vértigo y tinnitus en diferentes magnitudes. Un estudio retrospectivo mostró que cuando el tumor alcanza los tres centímetros aproximadamente, se comienza a ver un aumento del compromiso del nervio facial, con la parálisis consecuente ${ }^{3}$. Este cuadro clínico descrito en la literatura es bastante concordante con lo presentado por el paciente, siendo la fluctuación de los síntomas compatible con un hidrops secundario a la lesión. A pesar de que el hallazgo de un tumor de punta de peñasco ya había sido hecho previo a la consulta, la clínica ya orientaba a que esto podría corresponder a una causa tumoral de síndrome vertiginoso, que dado el compromiso cócleovestibular significativo, probablemente ya se encontraba en una etapa avanzada. De este modo es importante destacar la necesidad que dentro del diagnóstico diferencial de los síndromes vertiginosos periféricos se contemplen este tipo de cuadros, para que la solicitud de las neuroimágenes sea oportuna, permitiendo intervenir en forma adecuada para minimizar la morbilidad de cualquier tratamiento quirúrgico.

A pesar de la ya mencionada importancia de la clínica en el diagnóstico de los TSE, la TAC y la RNM son fundamentales para su completa evaluación, permitiendo proponer el adecuado diagnóstico diferencial y observar las características anatómicas necesarias para un adecuado abordaje quirúrgico. Ambos estudios muestran la ubicación retrolaberíntica característica, y las distintas estructuras comprometidas por el tumor (laberinto, cóclea, óseo, fosa posterior, etc), además de hallazgos específicos de cada método por separa$\mathrm{do}^{1,4}$. La TAC muestra con mayor precisión el compromiso óseo, típicamente centrado en la superficie posteromedial del hueso petroso, en forma anteromedial al seno sigmoideo, con destrucción de la pared posterior del hueso temporal, y calcificaciones intratumorales. En la RNM, la imagen característica revela una lesión con áreas hemorrágicas o quísticas hiperintensas tanto en T1 como en T2. En caso de necrosis o calcificaciones se pueden ver imágenes centrales hipointensas. Al contrastar con gadolinio casi todos los tumores dan una señal heterogénea en T1. Estas imágenes características descritas en la literatura son un sustento importante para postular el diagnóstico de TSE en este paciente, especialmente por la velocidad de progresión que se ve en los distintos estudios. En consecuencia, el seguimiento del paciente, deberá contar con la toma de neuroimágenes en forma periódica, a la espera del momento oportuno para tomar una conducta más agresiva.

Tal como se ha sugerido anteriormente, el tratamiento de elección para esta patología es la cirugía con resección total de la lesión previa biopsia de calidad que certifique el diagnóstico ${ }^{1,5}$. En la práctica, el nivel de compromiso óseo que generalmente presentan hace difícil una resección completa. Además no es infrecuente su extensión al ángulo pontocerebeloso, foramen yugular $u$ oído medio y externo, con casos avanzados que pueden llegar a comprometer fosa media craneana, clivus y esfenoide. La preservación de la audición debe ser un objetivo primordial de la cirugía, en especial en los casos de TSE bilaterales (más frecuentemente asociados a $\mathrm{VHL})^{3}$, reportando algunas series tiempo libre de enfermedad de más de 12 años luego de una resección completa 5 . Afortunadamente, el compromiso de este paciente es unilateral con lo cual hasta en el escenario más pesimista, el paciente quedaría con un nivel aceptable de incapacidad auditiva posterior a una cirugía, en comparación con el riesgo importante de compresión de estructuras vitales del Sistema Nervioso Central. Lamentablemente, la quimioterapia y radioterapia no han mostrado utilidad alguna en el tratamiento de los TSE, sólo habiendo un rol discreto de la radioterapia estereotáxica neoadyuvante en la disminución del sangrado intraoperatorio ${ }^{5}$.

\section{CONCLUSIONES}

Los TSE son lesiones que se diagnostican cada vez con más frecuencia, por lo que el conocimiento de su evolución clínica y manejo cobra cada vez más 
importancia para el otorrinolaringólogo. A pesar de tener una incidencia baja, los métodos diagnósticos actuales permiten detectarlo con mayor precocidad, lo cual conlleva a una gran responsabilidad.

日 caso clínico presentado cuenta con una serie de elementos clínicos y radiológicos que hacen del TSE la primera opción de diagnóstico. Lamentablemente el tejido obtenido para la biopsia no correspondió a la lesión misma, siendo probablemente una reacción inflamatoria perilesional. De este modo, una certificación histológica del diagnóstico parece ser fundamental para poder plantear una alternativa de manejo acuciosa. Ante la negativa de la paciente por someterse a un nuevo procedimiento, el seguimiento parece ser el método más adecuado para manejar a esta paciente, esperando el momento oportuno para tomar una decisión más agresiva en conjunto con el equipo de neurocirugía.

\section{BIBLIOGRAFÍA}

1. RODRIGUES S, FAGAN P, TURNER J. Endolymphatic sac tumors: a review of the St. Vincent's hospital experience. Otol Neurotol 2004; 25(4): 599-603.

2. WiLLIAMSON RA, COKER NJ. Endolymphatic sac tumor in von Hippel-Lindau disease. Otol Neurotol 2003; 24(5): 832.

3. BambakidIS NC, MEGrian CA, Rataheson RA. Differential grading of endolymphatic sac tumor extension by virtue of von Hippel-Lindau disease status. Otol Neurotol 2004; 25(5): 773-81

4. BANGJUE A, WHITE A, OSULIVAN P, ATLAS MD. Endolymphatic sac tumor. Otol Neurotol 2005; 26(4): 819-20.

5. HASHIMOTO M, YOKOTA A, URASAKI E, IMADA $\mathrm{H}$, YAMAMOTO $H$. Surgical treatment of endolymphatic sac tumor with adjunctive stereotactic radiation therapy-case report. Neurol Med Chir (Tokyo) 2004; 44(11): 595-9. 